\title{
PREVALENCE OF PAIN ON PALPATION OF THE INFERIOR POLE OF THE PATELLA AMONG PATIENTS WITH COMPLAINTS OF KNEE PAIN
}

\author{
Leonardo Addêo Ramos, Rogério Teixeira de Carvalho, Emerson Garms, \\ Marcelo Schmith Navarro, Rene Jorge Abdalla, Moisés Cohen
}

doi: 10.1590/S1807-59322009000300009

Ramos LA, Carvalho RT, Garms E, Navarro MS, Abdalla RJ, Cohen M. Prevalence of pain on palpation of the inferior pole of the patella among patients with complaints of knee pain. Clinics. 2009;64(3):199-202.

CONTEXT AND OBJECTIVE: Patellar tendinopathy is a common condition in sports. It may occur at any location of the patellar tendon, but the most commonly affected area is the inferior pole of the patella. Among various diagnostic tests, the one most used is palpation of the inferior pole of the patella. The aim of this study was to investigate the prevalence of pain complaints among individuals with pathological knee conditions and to evaluate palpation of the inferior pole of the patella as a diagnostic test for patellar tendinopathy.

METHODS: Palpation of the patellar tendon was performed on 318 individuals who presented with knee-related complaints. Palpation was performed with the individual in the supine position and the knee extended. The age, gender, physical activity and labor activity of each individual were recorded at the time the symptoms appeared; the diagnosis was also recorded.

RESULTS: Of the total number of individuals evaluated, $124(39 \%)$ felt pain on palpation of the inferior pole of the patella. Of these, only $40(32.3 \%)$ received a diagnosis of patellar tendinopathy. We did not observe any difference with respect to gender and age distribution. When evaluating daily physical activity levels, however, we observed that individuals with pain on palpation of the inferior pole of the patella experienced more intense physical activity.

CONCLUSIONS: Palpation of the inferior pole of the patella is a diagnostic procedure with high sensitivity and moderate specificity for diagnosing patellar tendinopathy, especially among individuals who perform activities with high functional demands.

KEYWORDS: Patellar; Tendinopathy; Physical examination; Clinical test; Athletes.

\section{INTRODUCTION}

Patellar tendinopathy is a common condition in sports. Its prevalence is variable and may reach $50 \%$ in sports such as volleyball, which involve jumping and landing..$^{1-3}$

A large number of names for this condition can be found in the literature, such as jumper's knee, patellar tendinitis, enthesitis of the patellar tendon, and patellar tendinosis. Some histopathological studies, however, have observed a lack of intense inflammatory processes in such cases.

Sports Traumatology Center (CETE), Department of Orthopedics and Traumatology, Federal University of São Paulo(UNIFESP) - São Paulo/ SP, Brazil.

Tel.: 55115539.5090

Email: leo_ramos@hotmail.com

Received for publication on October 10, 2008

Accepted for publication on November 29, 2008
Therefore, some authors have judged it better to use the term tendinopathy of the patellar tendon.., 5

This condition may occur in any location of the patellar tendon, but the most commonly affected area is the inferior pole of the patella. It is characterized by pain in the anterior portion of the knee that worsens with physical activity in movements related to jumping, ascending and descending slopes, and long periods of knee flexion. ${ }^{5}$

The diagnosis is made from taking a history, physical examination and performing subsidiary tests. Among various diagnostic tests, the one most used is palpation of the inferior pole of the patella.

Palpation of the patellar tendon together with its origin in the patella is easy to perform because of its superficial position. Some authors have reported a close relationship between pain on palpation and the occurrence of patellar tendinopathy. Among athletes such as young basketball 
players, this maneuver generated pain in $68 \%$ of the patients with patellar tendinopathy. On the other hand, some individuals reported pain but had normal ultrasound examination results. ${ }^{6}$

It should therefore be determined whether palpation of the inferior pole of the patella is a reliable test for diagnosing patellar tendinopathy. Furthermore, the prevalence of pain complaints during this maneuver should be measured among individuals with pathological knee conditions.

The aim of this study was to investigate the prevalence of pain complaints among individuals with pathological knee conditions and to evaluate palpation of the apex of the patella as a diagnostic test for patellar tendinopathy.

\section{MATERIALS AND METHODS}

The investigation was designed as a cross-sectional study among athletes from different sports. All patients were seen between December 2005 and November 2006. Palpation of the patellar tendon was performed on 318 individuals who presented with knee-related complaints. The study included 218 men and 100 women, thus totaling 636 knees, and was approved by the committee for ethics in research at our institution.

The individuals included in this study were between 15 and 40 years old, with a mean age of 28 years. Individuals were excluded if they had a diagnosis of fracture or recent direct trauma at the site or an active infection close to the joint.

The age, gender, physical activity and labor activity of each individual were recorded at the time the symptoms appeared; the diagnosis was also recorded. The activity level was defined in accordance with Tegner's classification. ${ }^{7}$

\section{Palpation Assessment}

All of the subjects were examined by one individual (L.A.R.) board-certified by the Brazilian Society of Knee Surgery (SBCJ). The examiner was not aware of the diagnosis. Palpation was performed with the individual in the supine position and the knee extended. The upper pole of the patella was grasped to anteriorize the apex of the patella. Using finger pressure on the patellar tendon immediately below the inferior pole of the patella, the test response was obtained. Only responses of "yes" or "no" were considered (Figure 1). The level of pain was not measured during palpation. The procedure was performed first on the affected side and then on the contralateral side.

The individuals were grouped according to maneuver results, physical activity level, bilateralism, diagnosis and gender. Complementary exams used as different methods

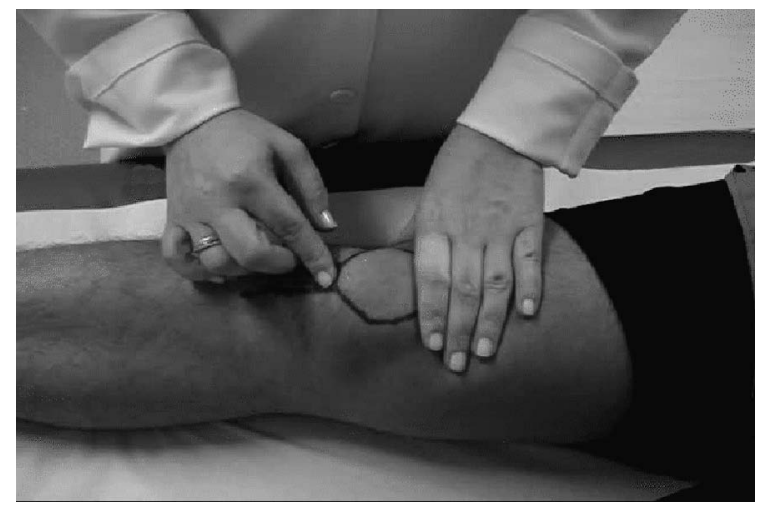

Figure 1 - Palpation of the inferior pole of the patella

for diagnosis were magnetic resonance imaging (MRI) and ultrasound (US). All imaging exams were analyzed by one senior board-certified musculoskeletal radiologist from the Radiology Department of the Federal University of São Paulo. The final diagnosis was made by one examiner (R.T.C.) from the Sports Traumatology Center (CETE).

\section{STATISTICAL METHODS}

Student's t test and the Fisher test were used to analyze the significance between groups. Results with $\mathrm{p}<0.05$ were deemed significant.

\section{RESULTS}

Of the total number of individuals evaluated, 124 (39\%) felt pain on palpation of the inferior pole of the patella. Of these, only $40(32.3 \%)$ received a diagnosis of patellar tendinopathy. Only one individual did not report any pain on palpation while presenting with a diagnosis of patellar tendinopathy. Therefore, we obtained a sensitivity of $97.6 \%$ and a specificity of $70 \%$ for this maneuver, with a positive predictive value of $67.53 \%$ and a negative predictive value of $97.85 \%$.

When comparing the individuals who presented with pain on palpation and those who did not, we observed that these groups were not different with respect to gender or age distribution. When evaluating daily physical activity levels, however, we observed that the individuals with pain on palpation of the inferior pole of the patella had more intense physical activity according to Tegner's classification. A comparison of the groups with respect to pain is presented in table 1.

The presence of pain in relation to diagnosis can be seen in table 2. There was a significant difference in the presence of pain between individuals with patellar tendinopathy and those with chondral lesions. 
Table 1 - Comparison of individuals with and without pain

\begin{tabular}{lcccc}
\hline & With pain & Without pain & total & \\
\hline Age & 28 & 29 & 28.57 & \\
Male & 85 & 133 & 218 & \\
Female & 39 & 61 & 100 & \\
Tegner & 5.6 & 4.4 & 4.8 & $\mathrm{p}<0.001$ \\
Contralateral & 50 & & 50 & \\
\hline Total & 124 & 194 & 318 & \\
\hline
\end{tabular}

Table 2 - Prevalence of pain according to diagnosis

\begin{tabular}{lccc}
\hline & total & with pain & $\mathrm{p}$ \\
\hline Patellar tendinopathy & 41 & 40 & $\mathrm{P}<0.001$ \\
ACL lesion* & 70 & 27 & \\
Meniscus lesion & 81 & 24 & \\
Chondral lesion & 23 & 3 & $\mathrm{p}=0.0132$ \\
Patellar-femoral syndrome & 40 & 17 & \\
Hamstring tendinopathy & 23 & 07 & \\
MCL lesion* & 16 & 01 & \\
Iliotibial friction syndrome & 08 & 02 & \\
PCL and PLC lesions* & 12 & 03 & \\
Chronic synovitis & 04 & 00 & \\
\hline
\end{tabular}

*ACL: anterior cruciate ligament; MCL: medial collateral ligament PCL: posterior cruciate ligament; PLC: Posterolateral corner

We observed the occurrence of pain in the contralateral knee in 50 individuals $(40 \%)$. This group was not differentiated from the remainder with respect to gender, age, diagnosis, or physical activity $(\mathrm{p}=0.83)$.

\section{DISCUSSION}

Pain on palpation of the apex of the patella has been considered to be an important preliminary diagnostic maneuver for patellar tendinopathy. The purpose of this study was to evaluate this maneuver among individuals who had sought specialized knee outpatient services so that we could observe the diagnostic relevance of this test.

Tenderness was the only sign present when evaluating patients with patellar tendinopathy. ${ }^{8}$ In another study, the prevalence of patellar tendinopathy among elite athletes was assessed using physical examination and anamnesis, and it was found that physical examination was the best test for estimating the prevalence of patellar tendinopathy. ${ }^{3}$

In our group of patients with a diagnosis of patellar tendinopathy, positive results were obtained from palpation of the apex of the patella in $97.5 \%$ of cases. When this maneuver was performed on all individuals who sought our outpatient services because of knee complaints, the prevalence of a positive test was $39 \%$. According to our findings among individuals with pain on palpation, the chance that this result could be related to patellar tendinitis was $67.5 \%$, giving a $32.5 \%$ chance of false positives. In the cases with negative results, the chance of false negatives was $2.15 \%$, since the negative predictive value was $97.85 \%$.

The specificity of this maneuver for diagnosing patellar tendinitis was $70 \%$, similar to others studies showing that this maneuver has moderate sensitivity and is nonspecific for patellar tendinopathy. ${ }^{6} \mathrm{We}$ considered that the sensitivity for this lesion in our study was high (97.6\%). This could be explained by the fact that we considered only the diagnosis that led the individuals to seek our outpatient service, thereby excluding individuals with degenerative alterations of the tendon and asymptomatic individuals.

With respect to differential diagnoses, we observed that palpation of the apex of the patella was positive for individuals with symptomatic lesions of the patellar tendon $(\mathrm{p}<0.001)$ and was inversely proportional for chondral lesions $(\mathrm{p}=0.0132)$.

Diagnoses of patellar tendinopathy based only on clinical data are unreliable and therefore must be viewed in the context of imaging examinations. Among athletic individuals, the presence of alterations on imaging examinations ranges from $18 \%$ to $29 \%$ in the literature. . $^{3,9,10,11}$ Ultrasound has better accuracy than magnetic resonance imaging. The specificity is similar between these methods, but ultrasound shows greater sensitivity ( $87 \%$ vs. $57 \%) .{ }^{12}$ Some authors believe, however, that there is little correlation between resonance and ultrasound findings. ${ }^{2,13,14}$

Painful manifestations and abnormalities in images may occur without any basis for such findings. There was a group of individuals who presented pain on palpation of the apex of the patella, and $84(68.5 \%)$ of these individuals did not receive a diagnosis of patellar tendinopathy. ${ }^{13,15}$

The painful tendons showed some alterations in colored ultrasound, and these individuals were practicing activities that were more intense than those who had normal examinations. ${ }^{16}$

Training errors are considered to be one of the main extrinsic predisposing factors, in addition to poor technique and inadequate athletic training equipment. Other intrinsic factors such as lack of flexibility and pronated feet are also involved. ${ }^{16,17}$

The average prevalence of this pathological condition in sports is $14 \%$, although some sports, such as volleyball, have a prevalence that may reach up to $45 \%$. $^{2,3}$ Sports that have shown greater prevalence of patellar tendinopathy include those involving high-intensity movements related to jumping and running, where the ground reaction force is transmitted 
directly to the extensor through eccentric movements that are 1.5 to two times more intense than the maximum concentric strength. $^{2}$ Among the risk factors we evaluated, there was a correlation with training level, which may represent the highly specific demands of different types of sports or training errors. ${ }^{2}$ The findings from this study demonstrate that individuals reporting pain on palpation of the patellar tendon had greater physical activity than those who had a negative test $(\mathrm{p}<0.0001)$.

Patellar tendinitis is frequently found bilaterally, and the prevalence of this condition between men and women has been found to be equal. Our results are in agreement with the findings of other authors. ${ }^{1,13}$

The main differential diagnosis for patellar tendinopathy is patellofemoral pain syndrome. The palpation maneuver is not specific enough to differentiate between these conditions, however, and was thus unable to provide a differential diagnosis when done in isolation (Table 2).

The drawback of this study was the inability to evaluate the patellar tendon in individuals with patellar pain, although it should be noted that this was not what made them seek medical assistance. Standardization of the palpation maneuver was another limitation, since there was no standard form for the force applied during palpation.
Nevertheless, we used the same standardization techniques used by other authors, which has shown good agreement between examiners. ${ }^{6}$ The level of pain could be graduated during palpation in order to improve the evaluation. ${ }^{6}$ Another limitation is related to Tegner's classification. This classification was introduced in 1985 with the objective of complementing the Lysholm classification. It attempts to discriminate between sedentary individuals and those who practice highly demanding activities. It is incapable, however, of differentiating between individuals who perform activities intensively and those who practice for only a few days per week. Moreover, it cannot differentiate between different ability levels. In order to minimize this problem, all evaluations using this classification were made by a single examiner. $^{7}$

\section{CONCLUSION}

We conclude that palpation of the inferior pole of the patella is a diagnostic procedure with high sensitivity and moderate specificity for diagnosing patellar tendinopathy, especially among individuals who perform activities with high functional demands.

\section{REFERENCES}

1. Ferretti A, Papandrea P, Conteduca F. Knee injuries in volleyball. Sports Med. 1990;10:132-8.

2. Lian OB, Engebrestsen L, Bahr R. Prevalence of jumper's knee among elite athletes from different sports. Am J Sports Med. 2005;33:561-7.

3. Lian O, Refsnes PE, Engebretsen L, Bahr R. Relationship between symptoms of jumper's knee and the ultrasound characteristics of the patellar tendon among high level male volleyball players. Scand J Med Sci Sports. 1996;6:291-6.

4. Khan KM, Maffulli N, Colemam BD, Cook JL, Taunton JE. Patellar tendinopathy: some aspects of basic science and clinical management. Br J Sports Med. 1998;32: 346-55.

5. Peers KH, Lysens RJ. Patellar tendinopathy in athletes: Current diagnostic and therapeutic recommendations. Sports Med. 2005;35:7187.

6. Cook JL, Khan KM, Kiss ZS, Purdam CR, Griffiths L. Reproducibility and clinical utility of tendon palpation to detect patellar tendinopathy in young basketball players. Br J Sports Med. 2001; 35: 65-9.

7. Tegner Y, Lysholm J. Rating systems in evaluation of knee ligament injuries. Clin Orthop Relat Res. 1985;198:43-9.

8. Duri ZA, Aichroth PM, Wilkins R, Jones J. Patellar tendonitis: clinical and literature review. Am J Knee Surg. 1999;12:99-108.

9. Cook JL, Khan KM, Kiss ZS, Griffiths L. Patellar tendinopathy in junior basketball players: a controlled clinical and ultrasonographic study of 268 patellar tendons in players aged $14-18$ years. Scand J Med Sci Sports. 2000;10:216-20.

10. Fredberg U, Bolvig L. Significance of ultrasonographically detected asymptomatic tendinosis in the patellar and Achilles tendons of elite soccer players: a longitudinal study. Am J Sports Med. 2002;30:48891.
11. Gisslèn K, Gyulai C, Soderman K, Alfredson H. High prevalence of jumper's knee and sonographic changes in Swedish elite junior volleyball players compared to matched controls. Br J Sports Med. 2005;39:298-301.

12. Warden SJ, Kiss ZS, Malara FA, Ooi AB, Cook JL, Crossley KM. Comparative accuracy of magnetic resonance imaging and ultrasonography in confirming clinically diagnosed patellar tendinopathy. Am J Sports Med. 2007;35:427-36.

13. Cook JL, Khan KM, Kiss ZS, Purdam CR, Griffiths L. Prospective imaging study of asymptomatic patellar tendinopathy in elite junior basketball players. J Ultrasound Med. 2000;19:473-9.

14. Khan KM, Visentini PJ, Kiss ZS, Desmond PM, Coleman BD, Cook JL, et al. Correlation of ultrasound and magnetic resonance imaging with clinical outcome after patellar tenotomy: prospective and retrospective studies. Clin J Sports Med. 1999;9:129-37.

15. Cook JL, Khan KM, Kiss ZS, Coleman BD, Griffiths L. Asymptomatic hypoechoic regions on patellar tendon ultrasound: a 4-year clinical and ultrasound follow-up of 46 tendons. Scand J Med Sci Sports. 2001;11: 321-7.

16. Gaida JE, Cook JL, Bass SL, Austen S, Kiss ZS. Are unilateral and bilateral patellar tendinopathy distinguished by differences in anthropometry, body composition, or muscle strength in elite female basketball players? Br J Sports Med. 2004;38:581-5.

17. Malliaras $\mathrm{P}$, Cook JL, Kent P. Reduced ankle dorsiflexion range may increase the risk of patellar tendon injury among volleyball players. J Sci Med Sports. 2006;4: 304-9. 